\title{
Aplicação das Cadeias de Markov no estudo do controle biológico da planta aquática Eichhornia azurea
}

\author{
Application of Markov Chain in the Study of Biological Control of Plant Aquatic \\ Eichhornia azurea
}

\author{
Ademir Amaro da Silveira Júnior ${ }^{1}$ e Graciele Paraguaia Silveira ${ }^{2}$ \\ ${ }^{1}$ Mestre - Universidade Federal de Goias, GO - Brasil \\ ademiramaro@yahoo.com.br \\ ${ }^{2}$ Doutor - Universidade Federal de Goias, GO - Brasil \\ gracimat@gmail.com
}

\begin{abstract}
Resumo
As plantas aquáticas exercem papel importante nas represas, pois auxiliam na filtração da água retirando alguns metais pesados, servindo de alimentos para peixes e de abrigo para alguns insetos. Entretanto, algumas espécies vêm se alastrando e causando grandes transtornos à população ribeirinha, usinas hidrelétricas e outros indivíduos que dependem dos lagos para sobreviverem. O propósito deste trabalho foi fazer um estudo inicial do controle biológico da macrófita aquática Eichhornia azurea por meio da inserção do inseto predador Thrypticus sp., utilizando modelagem matemática e Cadeias de Markov de ordem 1. Para tanto, um breve histórico sobre a planta aquática e o inseto é apresentado, assim como conceitos relacionados às Cadeias de Markov e suas aplicações na biomatemática são abordados, fornecendo subsídios para a realização da modelagem do problema. Dados reais obtidos de uma pesquisa desenvolvida pelo autor entre 2002 e 2003, no município de Coxim-MS, foram incorporados no processo de modelagem. Simulações de diferentes cenários foram realizadas, supondo infestação pela planta em represas interligadas e os resultados mostraram a quantidade de insetos necessários para controle em cada ciclo. O uso da modelagem matemática e das Cadeias de Markov permite a implementação de vários testes. Contudo, para se alcançar resultados mais precisos em relação ao controle biológico pretende-se, em trabalhos futuros, usar um modelo presa-predador e analisar com mais detalhes o problema, acrescentar mais hipóteses, além de discutir os aspectos referentes à população do predador no ambiente em questão. O trabalho é um fruto do Mestrado Profissional e foi escrito com a intenção de que docentes e estudantes do ensino médio possam compreender as resoluções dos exemplos apresentados.
\end{abstract}

Palavras-chave: Modelagem Matemática, Cadeias de Markov, Matemática Básica, Planta Aquática, Controle Biológico.

\begin{abstract}
The aquatic plants play an important role in the dams, because help in the water filtration by removing some heavy metals, serving of fish food and shelter for some insects. However, some species are spreading and causing great inconvenience to the local population, hydroelectrics and other individuals who depend on the lakes for survival. The purpose of this work was to make an initial study of the biological control of the aquatic macrophyte Eichhornia azurea by inserting the insect predator Thrypticus sp., using mathematical modeling and Markov chains of order 1. For that, a brief historical of the aquatic plant and of the insect is presented, as well as concepts related to Markov chains and their applications in biomathematics are discussed, supporting the realization of the modeling of the problem. Actual data obtained from a survey conducted by the author between 2002 and 2003, in the city of Coxim-MS, were incorporated in the modeling process. Simulations were performed for different scenarios, assuming infestation by plant in interconnected dams and the results showed the amount of insects needed to control in each cycle. The use of mathematical modeling and Markov chains allows the implementation of several tests. Nevertheless, to achieve more accurate results in relation to biological control we intend, in future work, to use a predator-prey model and analyze in more detail the problem, add more hypotheses, and to discuss aspects related to the predator population in environment in question. The work is a result of the Professional Masters and was written with the intention that teachers and high school students are being able understand the resolutions of the examples presented.
\end{abstract}

Keywords: Mathematical Modeling, Markov Chains, Basic Math, Aquatic Plant, Biological Control. 


\section{Introdução}

O estudo das interações inseto/planta tem despertado o interesse de pesquisadores nos últimos anos, pois a interação entre eles está relacionada à sua aplicação econômica através do controle de pragas e ecológica. A pesquisa sobre controle de pragas ocorre devido à crescente preocupação com o impacto ambiental causado por resíduos de pesticidas e agrotóxicos empregados nas plantações e rios infestados por plantas e insetos.

Muitos fazendeiros usam pesticidas para combater as infestações de insetos em suas plantações, assim como em plantas que infestam lagos, lavouras e pastos. Estas infestações trazem grande prejuízo ao produtor e ao meio ambiente.

Um dos problemas causados por pragas que podemos citar é a infestação de plantas aquáticas em lagoas ou represas.

Existem diferentes métodos para se obter um controle da proliferação de pragas. Um deles é o controle biológico, onde se usam insetos predadores para eliminar parte da praga. $\mathrm{O}$ controle biológico, como conservação de inimigos naturais ou liberação de inimigo natural, pode suprimir temporariamente pragas das culturas, seja nativo ou invasivo. Estes métodos fazem sentido quando o controle de pragas é necessário apenas em um local e em um momento específico. Portanto, o produtor interessado cobre o custo de implementação de tais práticas, a fim de reduzir as perdas financeiras devido aos danos causados pelas pragas.

O método adotado para o combate de infestações deve ser útil e eficiente no que diz respeito aos custos com o controle. Logo ele deve reduzir as perdas e ser mais conveniente e econômico do que outros métodos de controle disponíveis. Consequentemente, deve-se simular várias situações para que o custo não seja tão elevado.

Como existe uma interação entre os insetos e as plantas, pode-se aplicar o controle biológico como método a ser utilizado para tal necessidade, tendo em vista que os herbívoros ocupam o segundo nível trófico da cadeia alimentar [Begon(1996)].

Alguns autores acreditam que essas plantas são raramente consumidas quando vivas, sendo funcionalmente insignificantes para a cadeia alimentar aquática [Lodge(1991)].

No entanto, [Newman(1991)] considerou possível que o consumo de macrófitas aquáticas vivas pelos herbívoros seja subestimado. Esta hipótese é reforçada por autores como [Jacobsen e Sand-Jensen(1992)], e [Neiff e Poi de Neiff(1984)].

O Thrypticus sp. foi reconhecido como um agente de controle biológico desde levantamentos iniciais durante a década de 1960 realizados por [Bennett e Zwölfer.(1968)],

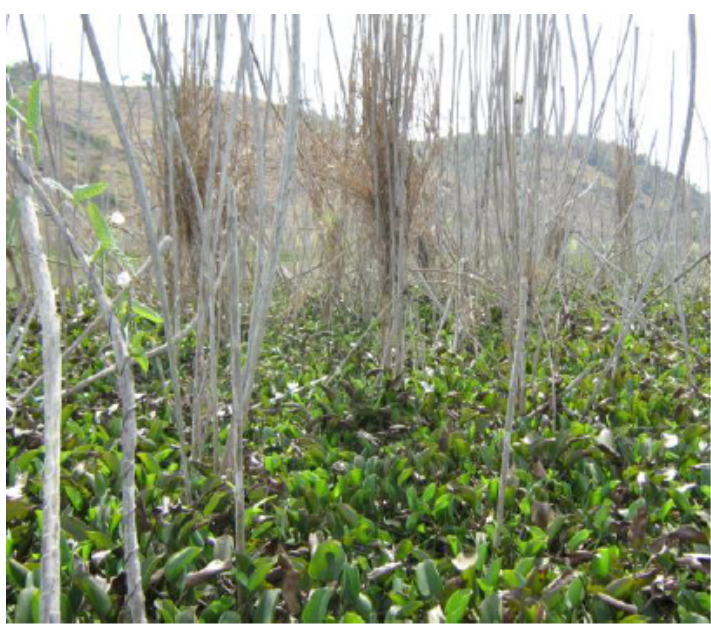

Figura 1: Reservatório de Aimorés-MG.

onde foi mencionado Thrypticus atacando jacinto de água no norte da América do Sul e Trinidad. Esses autores descreveram e ilustraram os danos e a estimada densidade de insetos, mas eles não deram informações específicas.

[Cruttwell(1973)] relatou investigações preliminares sobre a história de vida de uma espécie não identificada de Thrypticus associada com aguapé. Explorações de campo realizadas por uma equipe da América do Sul do Laboratório de Controle Biológico (SABCL) na Argentina entre 1997e e1999, mostraram que Thrypticus sp. atacou todas as espécies comuns de Pontederiaceae [Cordo e Hernandez(1999)], onde as larvas atacam na parte basal dos longos pecíolos de aguapé.

Na Figura 1 observa-se um reservatório na cidade de Aimorés-MG, infestado por uma planta aquática. As populações de macrófitas aquáticas formam extensas e densas colonizações.

A alta concentração destas macrófitas pode alterar os padrões de qualidade da água, como a concentração de oxigênio dissolvido, metilação do mercúrio, formação de gases tóxicos e outras [Mauro(1999)], [Carnwall(2005)], [Guimarães(2004)], promover condições adequadas para instalação e desenvolvimento de populações de organismos indesejáveis como insetos, moluscos e outros vetores de doenças humanas e animais [Forattini(1962)]; aumentar as perdas d'água por evapotranspiração no caso de macrófitas emergentes como a Eichhornia azurea, flutuante ou marginais, afetar a geração de energia elétrica, reduzir a capacidade de transporte de canais de irrigação e drenagem.

Pode-se citar ainda os prejuízos à produção e qualidade dos peixes, dificuldades na navegação, redução na capacidade de armazenamento de represas rurais e interferem na captação de água para irrigação e uso público, proporcionam prejuízos ao transporte fluvial e à edificações no corpo hídrico, especialmente pontes 


\section{[Vellini(2000)].}

Por outro lado, a presença das macrófitas aquáticas em pequenas quantidades é útil aos ambientes aquáticos, tendo em vista que filtram a água e servem de alimento para alguns peixes. Portanto, o objetivo não é o extermínio da planta e sim um controle do seu crescimento, para evitar os problemas já comentados.

Existem várias maneiras de se controlar o crescimento das macrófitas aquáticas. Entre elas pode-se citar a correção do ambiente, inimigos naturais, controle químico e mecânico, além de métodos de inundação [Pitelli(2000)]. No entanto, a aplicação dessas técnicas muitas vezes esbarra nas limitações ecológicas, sociais e econômicas [Vellini(2000)] recaindo em gastos elevados.

Mesmo que o controle biológico da planta aquática apresente alguns aspectos negativos, como a demora na obtenção dos resultados e o alto custo inicial, sua aplicação apresentou resultados satisfatórios em inúmeras situações, como no controle de Salvinia spp. na Austrália, Alternanthera philoxeroides em Porto Rico e Eichhornia crassipes no Sudão. Estes resultados mostram uma boa aceitação quanto ao emprego de tal prática.

$\mathrm{O}$ inseto identificado como um dos predadores da planta aquática é conhecido como Thrypticus sp., pertence a família Dolichopodidae e a ordem Díptera. Ele é conhecido como um predador da planta aquática desde a década de 1960 e portanto constitui-se em um grande candidato a agente no controle biológico destas plantas.

O objetivo deste trabalho foi tratar do estudo do controle biológico da planta aquática Eichhornia azurea, utilizando modelagem matemática e Cadeias de Markov de ordem 1. As Cadeias de Markov de ordem 1 envolvem probabilidades do estado atual para se obter resultados do estado posterior. No processo são necessárias multiplicação entre matrizes e vetores.

Este estudo foi realizado durante o mestrado profissional em matemática (PROFMAT) e por essa razão optou-se por um tema que faz parte do conhecimento dos alunos nos estados de Goiás e Mato Grosso do Sul. A escolha dos métodos se deveu ao fato de o trabalho também constituir uma proposta de ensino, possível de ser desenvolvida com alunos do Ensino Médio.

\section{Material}

A aplicação da estratégia controle biológico da planta aquática exige que primeiramente se faça um levantamento de seus predadores.

Em pesquisas realizadas anteriormente no ano de 2002 e 2003 [Silveira Jr e Vieira(2003)], estabeleceu-se os níveis de abundância de larvas de Thrypticus sp. encontrados nos pecíolos de Eichhornia azurea ocorrentes no município de Coxim-MS. Durante o levantamento da abundância predadores da planta aquática, foram amos-
Tabela 1: Entomofauna encontrada nos pecíolos de Eichhornia azurea Sw. (Kunth) ocorrentes no município de Coxim/MS 2002 e 2003.

\begin{tabular}{llc}
\hline \multicolumn{2}{c}{ Classificação } & \\
\cline { 1 - 2 } ORDEM & FAMÍLIA & INDIVÍDUOS \\
\hline Diptera & Dolichopodidae & 42 \\
Diptera & Cecidomyiidae & 05 \\
Diptera & Rhagionidae & 05 \\
Diptera & Simulíidae & 02 \\
Coleoptera & Curculionidae & 03 \\
Orthoptera & Sp1 & 03 \\
Thysanoptera & Phloethripidae & 51 \\
Hymenoptera & Mymaridae & 09 \\
TOTAL & 08 & 120 \\
\hline
\end{tabular}

tradas manchas de Eichhornia azurea ocorrentes numa lagoa permanente localizada no município de Coxim-MS ao final de cada mês. Nessas manchas foram retiradas cinco parcelas de $50 \times 50 \mathrm{~cm}$ de macrófita ao longo de um transecto estabelecido na área de maior densidade da mancha. Entre as parcelas foi mantida a distância de 2 metros. Todo material enquadrado nas parcelas foi recolhido em sacos plásticos, para triagem no laboratório.

No laboratório os pecíolos foram retirados, pesados para estimativa da biomassa a fresco e então colocados em frascos plásticos levemente umedecidos por um período de 30 dias, para obtenção dos insetos adultos cujas larvas se desenvolveram no interior destes pecíolos. Todos os insetos obtidos foram fixados em álcool $70 \% \mathrm{e}$ identificados os táxons do grupo família.

A biomassa total de pecíolos obtida nas coletas foi igual a 5212g. Quanto aos insetos coletados, foram obtidos 120 indivíduos pertencentes a 08 famílias. Das famílias obtidas mereceram destaque a família Phloeothrípidae (Thysanoptera) com 51 indivíduos e a família Dolichopodidae (Diptera) com 42 indivíduos, de acordo com a Tabela 1.

Nas plantas foram encontrados vários tipos de insetos, porém foi dado enfoque ao Thrypticus sp. (Diptera: Dolichopodidae) uma vez que esta mosca se alimenta do pecíolo da planta durante sua fase larval.

\section{Método: Modelagem Matemática e Cadeias de Markov}

A modelagem matemática estuda a simulação de sistemas reais a fim de prever o comportamento dos mesmos, sendo empregada em diversos campos, tais como física, química, biologia, economia e engenharias. Ou seja, modelagem matemática consiste em descrever matematicamente um fenômeno. 
Os modelos matemáticos apresentam uma série de aspectos úteis do ponto de vista científico. Além de apresentar naturalmente uma linguagem concisa, que pode vir a facilitar sua manipulação, um modelo matemático traz também aspectos como a possibilidade de confirmar ou rejeitar determinadas hipóteses relacionadas a complexos sistemas, revelar contradições em dados obtidos e/ou hipóteses formuladas, prever o comportamento de um sistema sob condições não testadas ou ainda não "testáveis", dentre outros.

Por outro lado, quanto maior é a proximidade do modelo com a realidade, mais complexo será o modelo. Isto significa um maior número de parâmetros e consequentemente uma maior dificuldade tanto na obtenção de dados a partir do modelo quanto na interpretação desses dados gerados pelo modelo em questão.

Segundo Howard Emmons [De Vries(2001)], não devemos produzir modelos descritivos mais compreensíveis quando utilizamos modelagem matemática, mas sim desenvolver modelos suficientemente simples que incorporam as principais características do fenômenos em discussão. Portanto, a modelagem matemática ajuda a evitar ou reduzir a necessidade de gastos excessivos em experimentos, ou até mesmo simular experimentos impossíveis de serem realizados na prática.

Para [Bassanezi(2011)], a modelagem matemática é a arte de transformar problemas reais em matemáticos e resolvê-los interpretando suas soluções na linguagem do mundo real.

A modelagem alia teoria e prática, motiva o usuário a buscar o entendimento da realidade que o cerca e buscar meios para agir sobre ela e transformá-la.

A modelagem matemática de fenômenos biológicos ganhou credibilidade com os modelos de interação entre espécies devidos a Lotka-Volterra e Kostitizin e com os modelos de epidemiologia de Kermack-McKendrick nos meados do século XX. A Biomatemática, que associa a biologia e a matemática, é uma área em ascenção, onde os pesquisadores se dedicam ao estudo de aplicações da matemática em problemas biológicos. Nos últimos anos a Biomatemática tem se desenvolvido graças a novas teorias tais como, Teoria Fuzzy, Espaços de Aspectos, Teoria do Caos, etc. [Bassanezi(2011)].

As Cadeias de Markov são um tipo especial de processo no qual utilizamos matrizes para otimizar resultados. O matemático Andrey Markov em 1906 conseguiu os primeiros resultados para estes processos.

Atualmente, as Cadeias de Markov têm sido estudadas e utilizadas em diversas áreas do conhecimento como, por exemplo, ciências biológicas, sociais e administrativas, probabilidades ligadas a jogos e evolução de populações.

Os cálculos em probabilidade levantam um questionamento quanto a existência ou não da dependência em ocorrências de fenômenos simultâneos ou sucessivos.
Partindo destes questionamentos sobre a teoria de probabilidades é que muitos matemáticos como, Thomas Bayes, Kolmogorov, Fisher, Pearson, foram em busca de respostas a perguntas similares, e com isso contribuíram no desvendar desse fascinante universo das incertezas.

Em particular, pode-se citar Andrei A. Markov, precursor no estudo da propriedade da perda de memória, propriedade que levou ao desenvolvimento da teoria sobre Cadeias de Markov, ferramenta de grande aplicabilidade nos mais diversos ramos da ciência.

Cadeias de Markov são os mais simples modelos matemáticos para os fenômenos aleatórios que evoluem no tempo. Sua estrutura simples torna possível dizer muito sobre o seu comportamento. Ao mesmo tempo, a classe das Cadeias de Markov é rico suficiente para servir em diversas aplicações [Norris(1998)].

Muitos fenômenos que ocorrem na natureza e na sociedade podem ser estudados, pelo menos em uma primeira aproximação, como se os fenômenos passassem a partir de um estado inicial, por uma sequência de estados, onde a transição de um estado para o seguinte, ocorre segundo uma certa probabilidade. No caso em que esta probabilidade de transição depende apenas do estado em que o fenômeno se encontra e do estado a seguir, o processo é denominado de Processo de Markov e uma sequência de estados envolvida nesse processo é denominada de Cadeias de Markov[Boldrini(1980)].

As Cadeias de Markov envolvem uma matriz, denominada matriz de transição, cujos elementos são as probabilidades de transição de um estado para outro. Para resolver um problema usando as Cadeias de Markov o diagrama de transição (ver Figura 2) tem o objetivo de facilitar na obtenção da matriz de transição.

O diagrama de transição é uma representação gráfica das Cadeias de Markov. Neste diagrama são visualizados os estados, representados por círculos, e as probabilidades de transição entre os estados. A Figura 2 mostra um diagrama de transição com 3 estados.

A gama de aplicações dos modelos que envolvem as Cadeias de Markov é verdadeiramente grande. Elas incluem praticamente qualquer sistema dinâmico cuja evolução ao longo do tempo envolve incerteza, desde que o estado do sistema seja adequadamente definido.

Primeiramente consideremos as Cadeias de Markov em que as mudanças de estado em determinados instantes de tempo $n$ seja denotado por $X_{n}$ e que pertença a um conjunto $S$ de estados possíveis, chamado espaço de estado.

Vamos supor que $S=\{1, \ldots, M\}$, para algum inteiro positivo $m$. As Cadeias de Markov são descritas em termos das probabilidades de transição $p_{i j}$.

Matematicamente,

$$
p_{i j}=P\left(X_{n+1}=j \mid X_{n}=i\right), \quad i, j \in S .
$$




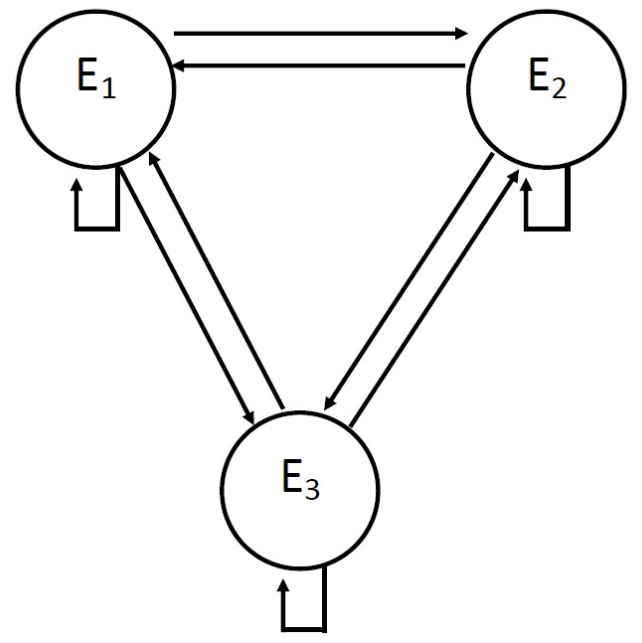

Figura 2: Diagrama de Transição com 3 estados.

O pressuposto fundamental dos processos de Markov é que as probabilidades de transição $p_{i j}$ são aplicadas sempre que voltar ao estado inicial, não importa o que aconteceu no passado. Portanto a probabilidade de ocorrer $X_{n+1}$ depende somente de $X_{n}$.

De acordo com [Bertsekas e Tsitsiklis(2000)] um modelo de Cadeias de Markov é especificado por meio da identificação:

(a) conjunto de estados $S=\{1, \ldots, M\}$.

(b) conjunto de transições possíveis, ou seja, os pares $(i, j)$, para que $p_{i j}>0$.

(c) os valores numéricos desses $p_{i j}$ são positivos.

As Cadeias de Markov especificadas são uma sequência de variáveis aleatórias $X_{0}, X_{1}, X_{2}, \ldots$, que tomam valores em $S$ e que satisfaz

$$
P\left(X_{n+1}=j \mid X_{n}=i, X_{n-1}=i_{n-1}, \ldots, X_{0}=i_{0}\right)=p_{i j}
$$

para todo $n$, todos os estados $i, j \in S$ e todas as possíveis sequências $i_{0}, \ldots, i_{n-1}$ de estados de anteriores.

Todos os elementos de um modelo de Cadeias de Markov podem ser escritos como uma matriz de probabilidade, que é simplesmente uma matriz bidimensional cujo elemento na $i$-ésima linha e j-ésima coluna é $p_{i j}$.

Assim,

$$
\left(\begin{array}{cccc}
p_{11} & p_{12} & \ldots & p_{1 m} \\
p_{21} & p_{22} & \ldots & p_{2 m} \\
\vdots & \vdots & \vdots & \vdots \\
p_{m 1} & p_{m 2} & \ldots & p_{m m}
\end{array}\right)
$$

Com a finalidade de agilizar o entendimento dos processos é de grande valia utilizar grafos para expor

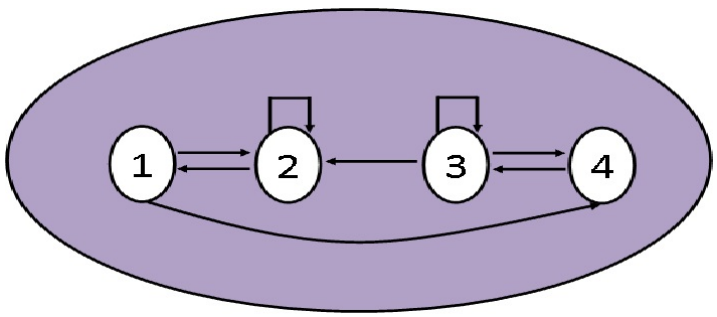

Figura 3: Grafo de transição de probabilidade.

o modelo da probabilidade de transição. A Figura 3 apresenta um exemplo de grafo, cujos nós $\{1,2,3,4\}$ são os estados e os arcos (setas) são as possíveis transições.

Neste trabalho usamos as Cadeias de Markov de ordem 1 , por envolver multiplicação de matrizes e vetores e uma estrutura possível de ser abordada no Ensino Médio.

\section{Resultados}

O cenário considerado consiste de uma situação em que três represas estão interligadas por córregos, onde há um fluxo de água entre elas, conforme ilustra a Figura 4. De posse dos dados levantados, elaboramos modelos matemáticos para otimizar, hipoteticamente, o número de insetos predadores à ser inserido em cada represa. Um estudo como esse é um primeiro passo importante, para tratar de um problema essencial para a qualidade de vida e preservação do meio ambiente.

As Cadeias de Markov de ordem 1 foram utilizadas em simulações de situações envolvendo as plantas e os insetos, onde supomos apenas um ciclo de vida de tais insetos.

O ciclo completo dos insetos pode levar semanas, meses ou mesmo anos. Contudo, é habitual usar uma única geração, como a unidade básica de tempo ao tentar gravar um modelo para o crescimento da população de insetos. Vários estágios do ciclo de vida podem ser representados escrevendo várias equações de diferenças [Edelstein-Keshet(1998)].

Segundo o biólogo e professor da Universidade Estadual de Mato Grosso do Sul, Dr. Gustavo H. C. Vieira, a infestação de lagos por macrófitas aquáticas depende de vários fatores, como o nível de claridade, nutrientes na água, abundância de predadores, etc., porém muitas vezes se considera tal infestação quando a área ocupada pelas macrófitas atinge $70 \%$ da área do lago.

Portanto, supomos que os lagos estão com 100\% de sua área ocupada por macrófitas aquáticas e modelaremos situações que estude o controle de 30\% dessas plantas, obtendo uma taxa menor ou igual a 70\%.

Partindo da situação problema, para escrevê-la em equações matemáticas o primeiro passo é determinar 


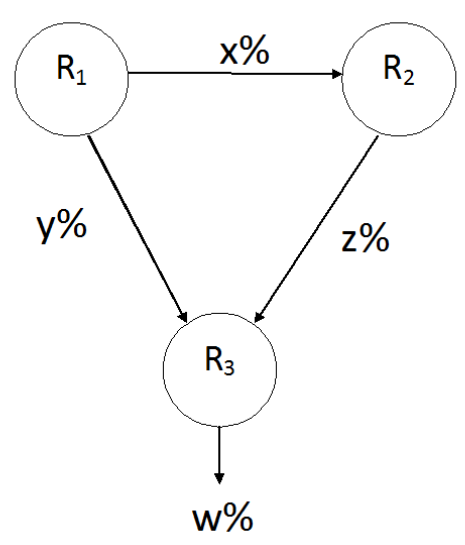

Figura 4: Representação das lagoas e fluxo de água.

quais são as grandezas conhecidas e desconhecidas envolvidas, para que possamos relacioná-las de alguma forma.

Considere o cenário em que três represas estão interligadas por córregos, com taxas de fluxos de água entre elas. Denotemos as represas por $R_{1}, R_{2}$ e $R_{3}$ e a partir das taxas obtemos a matriz que as representa. Em seguida, escrevemos o vetor que representa o número de insetos existentes inicialmente em cada lagoa e usamos o produto de matrizes para obter as equações necessárias.

Os cálculos são efetuados para analisar o que acontece com o número de insetos em cada represa, após vários ciclos de transferência de insetos.

Para cada represa, foi tomado o número de insetos predadores já existente e o número de insetos que desloca de um lago para outro através do fluxo da água.

Suponha que as represas estão interligadas por córregos, a represa $R_{1}$ deságua nas represas $R_{2}$ e $R_{3}$ a uma taxa de $x$ e $y$, respectivamente, a represa $R_{2}$ deságua na represa $R_{3}$ a uma taxa de $z$ e a represa $R_{3}$ deságua para o meio exterior EXT a uma taxa de $w$, conforme a Figura 4.

De acordo com isso, construímos a Tabela 2 apresentando as taxas de insetos que cada represa transfere para a outra juntamente com a planta que se desloca nas correntezas da água.

Tabela 2: Taxa de insetos transferido entre as represas.

\begin{tabular}{|c|c|c|c|c|}
\hline & $R_{1}$ & $R_{2}$ & $R_{3}$ & EXT \\
\hline \hline$R_{1}$ & $100-\mathrm{y}-\mathrm{x}$ & 0 & 0 & 0 \\
\hline$R_{2}$ & $\mathrm{y}$ & $100-\mathrm{z}$ & 0 & 0 \\
\hline$R_{3}$ & $\mathrm{x}$ & $\mathrm{z}$ & $100-\mathrm{w}$ & 0 \\
\hline EXT & 0 & 0 & $\mathrm{w}$ & 100 \\
\hline
\end{tabular}

A partir dos dados contidos na Tabela 2 aplicamos o princípio das Cadeias de Markov para obter o número de insetos em cada represa após um ciclo. Cada ciclo corresponde ao tempo necessário para que a taxa de transferência de insetos se efetue completamente, ou seja, as taxas de $x, y, z$ e $w$ passem de uma lagoa para a outra.

Para isso suponha o número inicial do insetos nas represas $R_{1}, R_{2}$ e $R_{3}$ como sendo $a, b$ e $c$ respectivamente e o do meio exterior como sendo $d$, logo obtemos as seguintes matrizes

$$
\begin{gathered}
\left(\begin{array}{cccc}
\frac{100-y-x}{100} & 0 & 0 & 0 \\
\frac{y}{100} & \frac{100-z}{100} & 0 & 0 \\
\frac{x}{100} & \frac{1}{100} & \frac{100-w}{100} & 0 \\
0 & 0 & \frac{w}{100} & 1
\end{array}\right) \\
\left(\begin{array}{c}
a \\
b \\
c \\
d
\end{array}\right) .
\end{gathered}
$$

Aplicando o produto das matrizes (1) e (2), segue que

$$
\left(\begin{array}{c}
\frac{a}{100}(100-y-x) \\
\frac{a}{100} y+\frac{b}{100}(100-z) \\
\frac{a}{100} x+\frac{b}{100} z+\frac{c}{100}(100-w) \\
\frac{c}{100} w+d
\end{array}\right)
$$

Assim, é possível calcular o número de insetos em cada represa após qualquer quantidade de ciclo, bastando aplicar novamente o produto da matriz (3) lograda no processo anterior pela matriz (1) inicial.

\section{Discussão}

Considere agora que os fluxos entre as represas sejam de $R_{1}$ para $R_{2}$ igual e $5 \%$, de $R_{1}$ para $R_{3}$ igual a $10 \%$, de $R_{2}$ para $R_{3}$ igual a $15 \%$ e de $R_{3}$ para o meio externo igual a $10 \%$ como apresenta a Figura 5. Então, aplicando novamente o procedimento, temos a matriz (4).

$$
\begin{aligned}
& \left(\begin{array}{cccc}
\frac{100-5-10}{100} & 0 & 0 & 0 \\
\frac{5}{100} & \frac{100-15}{100} & 0 & 0 \\
\frac{10}{100} & \frac{15}{100} & \frac{100-10}{100} & 0 \\
0 & 0 & \frac{10}{100} & \frac{100}{100}
\end{array}\right) \\
& \left(\begin{array}{cccc}
\frac{85}{100} & 0 & 0 & 0 \\
\frac{5}{100} & \frac{85}{100} & 0 & 0 \\
\frac{10}{100} & \frac{15}{100} & \frac{90}{100} & 0 \\
0 & 0 & \frac{10}{100} & \frac{100}{100}
\end{array}\right)
\end{aligned}
$$




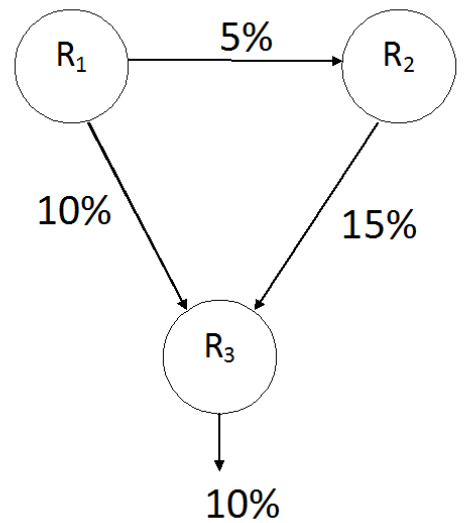

Figura 5: Representação das lagoas e fluxo de água.

De acordo com [Silveira Jr e Vieira(2003)], foi encontrado em uma área de $6,25 \mathrm{~m}^{2}$ o número de 42 Thrypticus sp., ou seja, o inseto predador da planta na fase larval.

Tome as represas $R_{1}, R_{2}$ e $R_{3}$ dispostas de acordo com a Figura 5 e com áreas iguais a $625 m^{2}, 312,5 m^{2}$ e $200 m^{2}$, respectivamente. Então, nas três represas tem-se uma quantidade de aproximadamente, 4200, 2100 e 1344, insetos já inseridos no meio em questão e zero insetos no meio exterior. Com isso, obtemos a matriz (5)

$$
\left(\begin{array}{c}
4200 \\
2100 \\
1344 \\
0
\end{array}\right)
$$

Aplicando o produto das matrizes (4) e (5), resulta:

$$
\begin{gathered}
\left(\begin{array}{c}
\frac{4200}{100}(85) \\
\frac{4200}{100}(5)+\frac{2100}{100}(85) \\
\frac{4200}{100}(10)+\frac{2100}{100}(15)+\frac{1344}{100}(90) \\
\frac{1344}{100}(10)
\end{array}\right), \\
\left(\begin{array}{c}
3570 \\
210+1785 \\
420+315+1210 \\
134 \\
\\
3570 \\
1995 \\
1945 \\
134
\end{array}\right)
\end{gathered}
$$

Ou seja, temos a represa $R_{1}$ com 3570 insetos, $R_{2}$ com 1995 insetos, $R_{3}$ com 1945 insetos e para o meio exterior seriam liberadom 134 insetos.
Assim, partindo da matriz (6) podemos estimar o número de insetos presentes em cada represa após qualquer quantidade de ciclos, bastando para isso aplicar novamente o produto da matriz (6) obtida no processo anterior pela matriz (4) inicial.

Como o objetivo foi conseguir uma maneira de estimar o número de insetos em cada represa para que haja um controle biológico da planta aquática, fizemos mais uma suposição. Baseado na pesquisa do autor em 2002 e 2003 , sabe-se que em $5212 \mathrm{~g}$ de pecíolos da macrófita aquática foram emcontrados 42 indivíduos do inseto Thrypticus sp., portanto temos uma taxa de $0,8 \%$ insetos para cada grama de pecíolo.

O número de insetos encontrados nos pecíolos, cuja taxa é de $0,8 \%$ insetos para cada grama de pecíolo não mantém controlada a quantidade de plantas nas lagoas, pois se tal fato ocorresse não teríamos o problema de infestação. Durante as pesquisas não encontramos autores que relatem sobre a quantidade de massa da planta ingerida pelo inseto durante sua fase larval e portanto vamos supor que este valor seja $2 \mathrm{~g}$.

Aplicamos o processo das Cadeias de Markov de ordem 1 para estimar o número de insetos que precisam ser inseridos na represa 1 de modo que esses insetos cheguem às represas 2 e 3 através do fluxo de água.

De acordo com os dados das modelagens, as represas possuem uma quantidade inicial de insetos predadores já conhecida. A área de cada represa e a massa de pecíolos da macrófica foram quantificadas no modelo.

Logo, verifica-se que a represa 3 possui área de $200 \mathrm{~m}^{2}$ e que em $6,25 \mathrm{~m}^{2}$ obtivemos $5212 \mathrm{~g}$ de pecíolos da macrófita aquática, então a represa 3 possui uma massa aproximada de $167 \mathrm{~kg}$ de pecíolos da planta aquática. Como foi mencionado anteriormente, o controle biológico deve ocorrer quando a área ocupada pela planta é maior que $70 \%$ da área da represa, então consideramos apenas uma massa de $50,1 \mathrm{Kg}$ que corresponde a $30 \%$ da área da represa. De modo análogo a represa 2 tem área de $312,5 m^{2}$ e portanto $261 \mathrm{~kg}$ de pecíolos, onde serão considerados para o controle apenas $78,3 \mathrm{~kg}$ e a represa 1 tem área de $625 \mathrm{~m}^{2}$ e contudo $522 \mathrm{~kg}$ de pecíolos, onde serão considerados $156,6 \mathrm{~kg}$.

Supondo um consumo de $2 \mathrm{~g}$ de pecíolo pelo inseto durante a fase larval, então para que haja um controle deve se ter um número de insetos que remova 30\% das macrófitas, ou seja, deve-se obter na represa 3 uma quantidade de 25.000 insetos, na represa 2 um total de 39.000 e na represa 1 uma soma de 78.000 insetos.

Para estimar a quantidade de insetos que devem ser inseridos na represa 1 , para que transcorridos vários ciclos, chegue a quantidade necessária na represa 3, outras simulações serão feitas usando as matrizes encontradas anteriormente. 


\subsection{Simulação}

As hipóteses consideradas no processo de modelagem basearam-se em dados reais, coletados no município de Coxim-MS. Partindo destes dados e juntamente com outras hipóteses que estão listadas a seguir, temos:

1. As represas $R_{1}, R_{2}$ e $R_{3}$ têm áreas de $150 m^{2}, 250 m^{2}$ e $80 m^{2}$, respectivamente;

2. As taxas de fluxo de deságua são de $6 \%$ de $R_{1}$ para $R_{2}, 4 \%$ de $R_{2}$ para $R_{3}, 3 \%$ de $R_{1}$ para $R_{3}$ e $5 \%$ de $R_{3}$ para o meio $E X T$;

3. Em cada $6,25 m^{2}$ obtemos 42 insetos predadores e $5212 \mathrm{~g}$ de pecíolos de planta aquática;

4. Cada larva é capaz de se alimentar com $2 \mathrm{~g}$ de pecíolo da planta aquática;

5. A taxa em que se considera a infestação é maior que $70 \%$ da área da represa;

6. As represas $R_{1}, R_{2}$ e $R_{3}$ estão infestadas de planta aquática com taxas de $85 \%, 90 \%$ e $100 \%$, respectivamente.

Logo, organizando os dados das represas $R_{1}, R_{2} \mathrm{e}$ $R_{3}$ tem-se a Tabela 3, onde $I_{0}$ é a quantidade de insetos contidos inicialmente, $M_{p}$ é a massa de pecíolo na lagoa, $i$ corresponde a taxa a ser combatida, $M_{c}$ é a massa a ser combatida e $I_{n}$ é a quantidade de insetos necessários.

Tabela 3: Dados para simulação.

\begin{tabular}{|c|c|c|c|c|c|}
\hline Represas & $I_{0}$ & $M_{p}(\mathrm{~kg})$ & $i(\%)$ & $M_{c}(\mathrm{~kg})$ & $I_{n}$ \\
\hline \hline$R_{1}$ & 1008 & 125 & 15 & 18,75 & 9.375 \\
\hline$R_{2}$ & 1680 & 208,5 & 20 & 41,7 & 20.850 \\
\hline$R_{3}$ & 538 & 66,7 & 30 & 20 & 10.000 \\
\hline
\end{tabular}

A Figura 6 representa o diagrama de transição desta situação hipotética.

A Tabela 4 apresenta as taxas de insetos transferidos entre as represas $R_{1}, R_{2}$ e $R_{3}$ na simulação 3 .

Tabela 4: Taxa de insetos transferidos entre as represas na simulação.

\begin{tabular}{|c||c|c|c|c|}
\hline & $R_{1}$ & $R_{2}$ & $R_{3}$ & EXT \\
\hline \hline$R_{1}$ & $100-6-3$ & 0 & 0 & 0 \\
\hline$R_{2}$ & 6 & $100-4$ & 0 & 0 \\
\hline$R_{3}$ & 3 & 4 & $100-5$ & 0 \\
\hline EXT & 0 & 0 & 5 & 100 \\
\hline
\end{tabular}

A matriz (7) é composta pelas taxas de transferências de insetos entre as represas na simulação e a matriz (8)

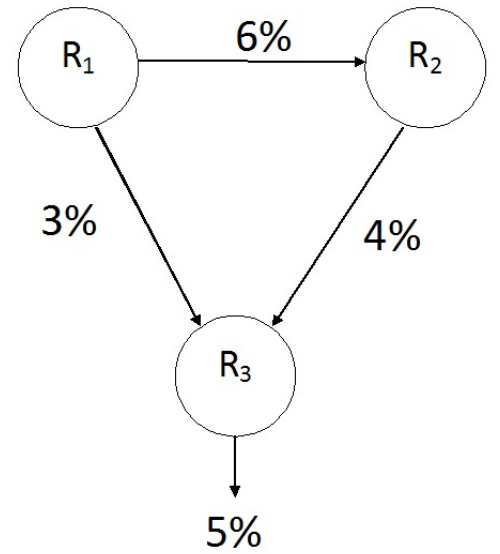

Figura 6: Representação das lagoas e taxa de fluxo de água na simulação.

representa o número de insetos contidos inicialmente nas represas

$$
\begin{gathered}
\left(\begin{array}{cccc}
\frac{91}{100} & 0 & 0 & 0 \\
\frac{6}{100} & \frac{96}{100} & 0 & 0 \\
\frac{3}{100} & \frac{4}{100} & \frac{95}{100} & 0 \\
0 & 0 & \frac{5}{100} & \frac{100}{100}
\end{array}\right) \\
\left(\begin{array}{c}
1008 \\
1680 \\
538 \\
0
\end{array}\right)
\end{gathered}
$$

Adicionando 8367 insetos na represa $R_{1}$ e aplicando o produto das matrizes (8) e (7), obtemos:

$$
\begin{gathered}
\left(\begin{array}{c}
\frac{9375}{100}(91) \\
\frac{9375}{100}(6)+\frac{1680}{100}(96) \\
\frac{9375}{100}(3)+\frac{1680}{100}(4)+\frac{538}{100}(95) \\
\frac{538}{100}(5)
\end{array}\right) \\
\left(\begin{array}{c}
8531 \\
2175 \\
860 \\
27
\end{array}\right)
\end{gathered}
$$

Isto é, após 1 ciclo, temos a represa $R_{1}$ com 8531 insetos, $R_{2}$ com 2175 insetos, $R_{3}$ com 860 insetos e para o meio exterior seriam liberados 27 insetos.

Nestas condições a represa 2 atinge o número máximo de insetos no ciclo 3 com a quantidade de 2297. Esta quantidade não é suficiente para que tenhamos um controle biológico na represa 2, deve-se inserir insetos nesta represa para completar a quantidade necessária. 


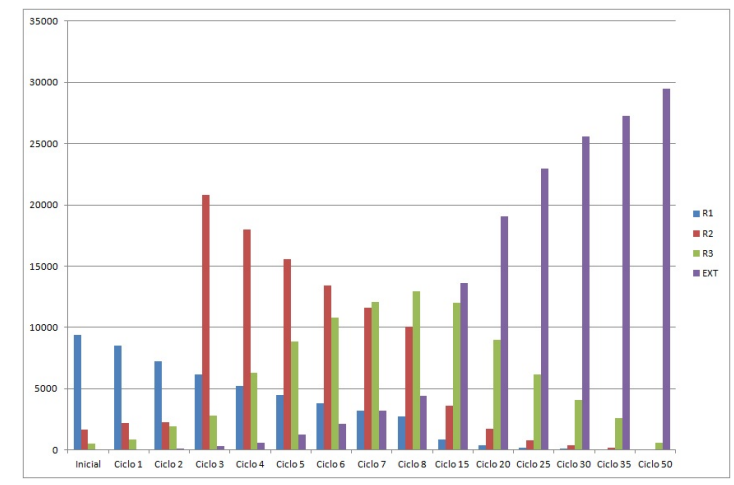

Figura 7: Quantidades de insetos por lagoa após vários ciclos.

Portanto, pode-se inserir 18.500 insetos no ciclo 3 para a obtenção do controle nesta represa.

Com isso, passaremos a ter a quantidade necessária para o controle na represa 2 e prosseguindo com os ciclos, obtém-se a quantidade necessária na represa 3 no ciclo 6 .

A Figura 7 mostra a situação, referente ao número de insetos em cada represa após vários ciclos.

Observando os dados no gráfico verificamos que os cálculos apontam uma tendência de eliminação total do inseto nestas lagoas, evitando assim uma infestação com o inseto. Entretanto, fazem-se necessários outros estudos mais detalhados para comprovar este fato.

\section{Conclusões}

O propósito principal deste trabalho foi mostrar como a modelagem matemática, que é um método usado por muitos pesquisadores entre as áreas de matemática e ecologia aplicada na resolução de problemas, pode ser inserida no processo de ensino e aprendizagem, por exemplo no ensino médio, permitindo que conteúdos matemáticos sejam aplicados na busca de soluções para situações reais. Para exemplificar, tratamos do estudo do controle biológico da planta aquática Eichhornia azurea.

Vários autores consideram as macrófitas aquáticas como de pouca importância enquanto vivas, para a cadeia alimentar aquática [Lodge(1991)], [Hutchinson(1975)], o que estaria relacionado à baixa qualidade nutricional dessas plantas, que geralmente apresentam uma alta concentração de celulose o que leva sua digestibilidade a ser muito baixa. Entretanto, verificamos que outros autores acreditam que o consumo de macrófitas aquáticas pelos insetos herbívoros seja subestimado [Newman(1991)].

Nos levantamentos realizados em macrófitas aquáticas Eichhornia azurea confirmou-se que alguns insetos predam a planta durante a fase larval, e um deles é conhecido como Thrypticus sp.. Existem outros insetos que vivem na planta, porém a utilizam apenas como abrigo e local de caça.

Quando há crescimento desordenado da planta aquática, uma das alternativas é o controle biológico, que deve ser realizado de modo que não prejudique o meio ambiente e os moradores que usam os lagos como fonte de vida, seja para a pesca ou para o uso da planta na fabricação de artesanatos.

A modelagem matemática é um método cada vez mais adotado, que encontra-se em grande expansão na matemática. Muitos problemas podem ser modelados e escritos na forma matemática, facilitando o entendimento e a análise de seus dados. Com a modelagem, numa situação real, é possível efetuar estudos a fim de minimizar custos operacionais, através da implementação de vários testes antes que se inicie os gastos.

As Cadeias de Markov foram o procedimento escolhido, para juntamente com a modelagem, apontar soluções para o problema proposto. Tal conceito é uma grande ferramenta nos estudos que envolvem probabilidades, sendo muito útil em diferentes áreas de estudo. A aplicação das Cadeias de Markov nas simulações produzidas permitiu-nos obter resultados coerentes e verificar que existem várias possibilidades de inserção de insetos, para combater o excesso da planta aquática. Na prática, a execução de vários testes demandaria muito tempo e dinheiro para serem analisados.

A união de problemas ambientais com a matemática constitui-se em uma maneira de avaliar parâmetros e predizer resultados, e isso pode ser abordado por professores nas escolas, inclusive como forma de chamar a atenção dos alunos para as questões ambientais e despertar atitudes.

Vale ressaltar que, para se obter resultados que possam efetivamente auxiliar os especialistas, com maior garantia de controle biológico da macrófita aquática Eichhornia azurea, utilizando ou não o inseto predador Thrypticus sp., deve-se pesquisar o tema com maior rigor e realizar outras simulações. Sugerimos alterar as áreas das lagoas, área ocupada pelas plantas aquáticas, avaliar a taxa de escoamento da água entre as lagoas, além de considerar a dinâmica vital do inseto, incorporando informações sobre nascimento, mortalidade e fatores externos que influenciam sua população.

Em trabalhos futuros, pretende-se analisar o problema usando modelo presa-predador, que é descrito por equações diferenciais.

\section{Referências}

[Bassanezi(2011)] Bassanezi, R. C. (2011). Ensinoaprendizagem com Modelagem Matemática: uma nova estratégia. Contexto. 
[Begon(1996)] Begon, J. e. T. C., M.; Harper (1996). Ecology: Individuals, Populations and Comunities. Blackwell Science Ltda.

[Bennett e Zwölfer.(1968)] Bennett, F. D., Zwölfer., H. (1968). Exploration for natural enemies of the Water hyacinth in northern South America and Trinidad. Hyacinth Control.

[Bertsekas e Tsitsiklis(2000)] Bertsekas, D. P., Tsitsiklis, J. N. (2000). Introduction to Probability.

[Boldrini(1980)] Boldrini, C. S. R. V. W. H., J.L. (1980). Álgebra linear. Haper e Row do Brasil.

[Carnwall(2005)] Carnwall, M. e. a. (2005). Avaliação do impacto da colonização de egeria densa sobre a variação dos concentrações de oxigênio dissolvido na água e sobrevivência de poecillia reticulata. Planta Daninha.

[Cordo e Hernandez(1999)] Cordo, A. J. S., H. A., Hernandez, M. C. (1999). The petiole mining by Thrypticus sp. (diptera: Dolichopodidae), a new agent for the biological control of water hyacinth (eichhornia crassipes). Em: Proceedings of the X International Symposium on Biological Control of Weeds.

[Cruttwell(1973)] Cruttwell, R. E. (1973). Preliminary investigations on some insects causing minor damage to Water hyacinth, Eichhornia crassipes.

[De Vries(2001)] De Vries, G. (2001). What is Mathematical Modelling. Department of Mathematical Sciences, University of Alberta.

[Edelstein-Keshet(1998)] Edelstein-Keshet, L. (1998). Mathematical Model in Biology. McGraw Hill.

[Forattini(1962)] Forattini, O. P. (1962). Entomologia médica. Editora Universidade de São Paulo.

[Guimarães(2004)] Guimarães, G. L. (2004). Impactos do controle de macrófitas aquáticas com o herbicida 2,4 d em mesocosmos. Tese de Doutorado, Universidade Estadual de Campinas.

[Hutchinson(1975)] Hutchinson, G. (1975). A treatise on limnology. Limnological Botany. Wiley-interscience.

[Jacobsen e Sand-Jensen(1992)] Jacobsen, D., SandJensen, K. (1992). Herbivory of invertebrates on submerged macrophytes from danish freshwaters. Freshwater Biology.

[Lodge(1991)] Lodge, M. (1991). Herbivory on freshwater macrophytes. Aquatic Botany.

[Mauro(1999)] Mauro, J. R. D. M. R., J. B. N.; Guimarães (1999). Aguapé agrava contaminação por mercúrio. Ciência Hoje.
[Neiff e Poi de Neiff(1984)] Neiff, J. J., Poi de Neiff, A. (1984). Cambios estacionales em la biomassa de Eichhornia crassipes (mart.) solms y su fauna em una laguna del chaco (argentina). Em: Ecosur.

[Newman(1991)] Newman, R. (1991). Herbivory and detritivory on freshwater macrophytes by invertebrates: a review. J. N. Am. Benthol. Soc.

[Norris(1998)] Norris, J. R. (1998). Markov Chains. University of Cambridge.

[Pitelli(2000)] Pitelli, R. e. M. D., R.A.; Pitelli (2000). Controle biológico de macrófitas aquáticas. Workshop Ecologia e Manejo de Macrófitas Aquáticas.

[Silveira Jr e Vieira(2003)] Silveira Jr, A., Vieira, G. (2003). Abundância da Larvas de Thrypticus sp. (Díptera: Dolichopodidae) em Pecíolos da Macrófita Aquática Eichhornia azurea sw. (Kunth) (Pontederiaceae) ocorrentes no município de Coxim/MS.

[Vellini(2000)] Vellini, E. (2000). Controle de Plantas Aquáticas. Workshop Ecologia e Manejo de Macrófitas Aquáticas. 